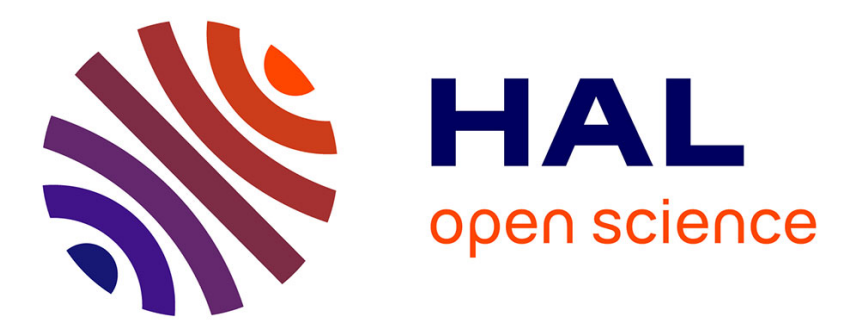

\title{
Transition from spin-orbit to hyperfine interaction dominated spin relaxation in a cold fluid of dipolar excitons
}

\author{
Ran Finkelstein, Kobi Cohen, Benoit Jouault, Ken West, Loren N. Pfeiffer, \\ Maria Vladimirova, Ronen Rapaport
}

\section{To cite this version:}

Ran Finkelstein, Kobi Cohen, Benoit Jouault, Ken West, Loren N. Pfeiffer, et al.. Transition from spinorbit to hyperfine interaction dominated spin relaxation in a cold fluid of dipolar excitons. Physical Review B, 2017, 96 (8), pp.085404. 10.1103/PhysRevB.96.085404 . hal-01577957v2

\section{HAL Id: hal-01577957 \\ https://hal.science/hal-01577957v2}

Submitted on 8 Sep 2017

HAL is a multi-disciplinary open access archive for the deposit and dissemination of scientific research documents, whether they are published or not. The documents may come from teaching and research institutions in France or abroad, or from public or private research centers.
L'archive ouverte pluridisciplinaire HAL, est destinée au dépôt et à la diffusion de documents scientifiques de niveau recherche, publiés ou non, émanant des établissements d'enseignement et de recherche français ou étrangers, des laboratoires publics ou privés. 


\title{
Transition from spin-orbit to hyperfine interaction dominated spin relaxation in a cold fluid of dipolar excitons
}

\author{
Ran Finkelstein, ${ }^{1,}{ }^{*}$ Kobi Cohen, ${ }^{1}$ Benoit Jouault,${ }^{2}$ Ken West,${ }^{3}$ Loren N. Pfeiffer ${ }^{3}$ Masha Vladimirova, ${ }^{2}$ and Ronen Rapaport ${ }^{1,4}$ \\ ${ }^{1}$ Racah Institute of Physics, The Hebrew University of Jerusalem, Jerusalem 9190401, Israel \\ ${ }^{2}$ Laboratoire Charles Coulomb, UMR 5221 CNRS-Université de Montpellier, Montpellier F-34095, France \\ ${ }^{3}$ Department of Electrical Engineering, Princeton University, Princeton, New Jersey 08544, USA \\ ${ }^{4}$ Department of Applied Physics, The Hebrew University of Jerusalem, Jerusalem 9190401, Israel
}

(Received 22 May 2017; published 2 August 2017)

\begin{abstract}
We measure the spin-resolved transport of dipolar excitons in a biased GaAs double quantum well structure. From these measurements we extract both spin lifetime and mobility of the excitons. We find that below a temperature of $4.8 \mathrm{~K}$ there is a sharp increase in the spin lifetime of the excitons, together with a sharp reduction in their mobility. Below a critical power the spin lifetime increases with increasing mobility and density, while above the critical power the opposite trend is observed. We interpret this transition as evidence of the interplay between two different spin dephasing mechanisms: at low mobility the dephasing is dominated by the hyperfine interaction with the lattice nuclei spins, while at higher mobility the spin-orbit interaction dominates and a Dyakonov-Perel spin relaxation takes over. The excitation power and temperature regime where the hyperfine interaction induced spin dephasing is observed correlates with the regime where a dark dipolar quantum liquid was reported recently on a similar sample.
\end{abstract}

DOI: 10.1103/PhysRevB.96.085404

\section{INTRODUCTION}

The spin degree of freedom plays a crucial role in electronic, magnetic, optical, and transport properties of semiconductor nanostructures, and spin is also one of the most promising candidates for encoding and transporting quantum information in such structures. The state of an electron spin is very sensitive to interactions with its environment. Two main interactions are known to affect electron spin: spin-orbit interaction, that couples the spin of electron to its motion, and hyperfine interaction, that couples electron spin to the spin of the lattice nuclei. They give rise to striking phenomena such as the spin Hall effect [1], a persistent spin helix [2], and self-sustained polarization oscillations [3], but at the same time they are the two main sources of spin relaxation and dephasing [4,5]. The relative importance of these two types of interaction is related to the electron mobility $[6,7]$.

This fact is particularly interesting for an electron bound in a dipolar, or indirect exciton (IX), a Coulomb-bound pair made of an electron and a hole, where each constituent is confined in a separate GaAs quantum well separated from the other by a thin $(\mathrm{Al}, \mathrm{Ga}) \mathrm{As}$ barrier. Such IXs form a long-living two-dimensional fluid of interacting bosonlike particles, with a complex, fourfold internal spin structure. Two of the IX spin states can couple to the light (bright states), they have spin projection along the growth axis $S_{z}= \pm 1$, while two other spin states $\left(S_{z}= \pm 2\right)$ are dark. It was predicted that Bose-Einstein condensation of the IX gas into the dark spin state should occur below a critical temperature [8] and that the condensed phase should be strongly correlated due to the strong dipolar interactions between the particles, similarly to a quantum liquid $[9,10]$. Recent experiments on dipolar IXs have reported a condensation to a dark, correlated quantum

\footnotetext{
*Present address: Department of Physics of Complex Systems, Weizmann Institute of Science, Rehovot 76100, Israel.
}

liquidlike phase [11-13], with a strongly quenched IX motion [12-14], as well as reports on a condensate with an extended spatial coherence [15-17] and a ballistic spin motion [18].

There are many open questions on the properties, the microscopic nature, and the formation dynamics of the newly observed collective phase. For example, the darkening of the IX fluid is suggested to be attributed to a spontaneous condensation of IX to the low-energy dark spin states $[8,11,13,17]$. In this context, the spin of the electron inside the IX could in principle be such a complementary, sensitive probe of IX dynamics. Indeed, long spin lifetimes (up to tens of nanoseconds) have been reported for IX [19-24]. This is due to dramatic reduction of the electron-hole exchange interaction within IX, which is responsible for the fast spin relaxation of traditional, direct excitons (DXs) in semiconductor quantum wells [25]. Because hole spin relaxation is usually much faster than spin relaxation of an electron, IX spin lifetime is determined by the constituent electron-spin lifetime. Thus, for IX spin relaxation we can expect a phenomenology similar to that of a two-dimensional electron gas. When IXs are localized (e.g., as observed in low-mobility correlated liquid of IXs, or in the case of strong disorder), the spin dephasing could be dominated by the hyperfine interaction via the fluctuations of the Overhauser field $B_{N}$ [3-5]. This mechanism is schematically represented in Fig. 1(d): electron-spin relaxation rate is determined by the electron correlation time $\tau_{c}$ (during which the Overhauser field experienced by an electron can be considered as constant) and the squared average value of $B_{N}$. In contrast, in a regime where IXs are highly mobile, fluctuations of the Overhauser field are averaged out. In this case electron-spin dephasing is mainly due to spin-orbit interaction via the Dyakonov-Perel (DP) mechanism [Fig. 1(c)] [26]. Here the relevant time is the momentum scattering time $\tau_{p}$.

A natural way to probe these two regimes is to study the correlation between IX spin lifetime and mobility by changing the lattice temperature and the exciton density. To get access to both spin lifetime and mobility, spatial extension of the 

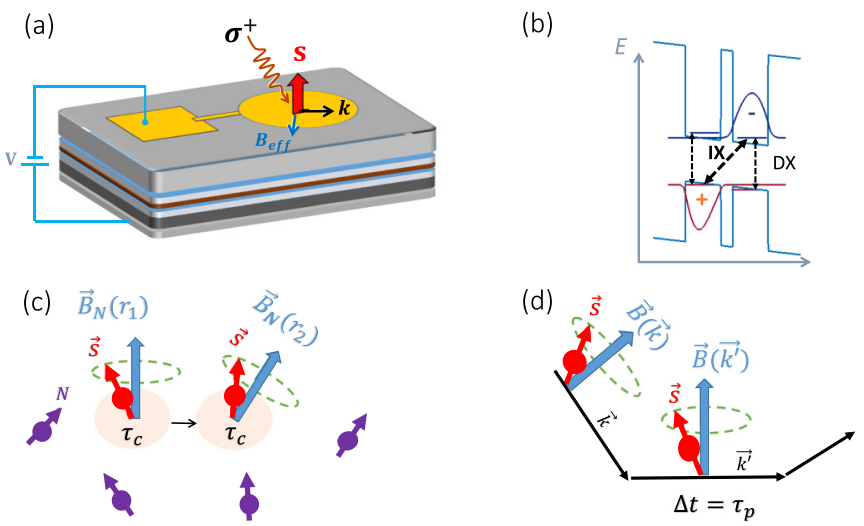

FIG. 1. (a) Sketch of the sample and experimental setup. Coupled quantum wells subjected to an electric field in the $z$ direction are excited with circularly polarized light resonant with the DX energy. (b) Band diagram of the biased GaAs/AlGaAs coupled quantum wells. A resonant excitation of the DX transition is followed by an electron tunneling across the barrier and the formation of IXs. (c) Hyperfine interaction-induced spin relaxation for localized excitons: relaxation rate is inversely proportional to the electron-spin correlation time $\tau_{c}$. This mechanism is quenched for mobile excitons. (d) Dyakonov-Perel spin-relaxation mechanism for moving excitons via fluctuations of the effective magnetic field induced by spinorbit interaction. Relaxation rate is proportional to the momentum scattering time $\tau_{p}$

IX cloud upon pointlike excitation and its polarization degree should be analyzed in the same experiment. Previous works have reported a significant decrease of the IX spin lifetime with increasing IX diffusivity, a behavior consistent with the DP relaxation mechanism of IX [20]. However, the role of the Overhauser field in the exciton spin dynamics has not been evidenced so far. Indeed, when the hyperfine mechanism of spin relaxation dominates, one should expect an increase of the IX spin lifetime with increasing IX diffusivity and density.

In this work we report a temperature and density regime in an excitonic system where a transition from a decreasing spin lifetime to an increasing spin lifetime with increasing mobility takes place. This suggests that hyperfine interaction does play a role in the IX spin relaxation when a transition from the high- to low-mobility state of the IX fluid occurs. Remarkably, the transition temperature between the two regimes is found to closely match the previously reported critical temperature for the condensation of an IX liquid.

The paper is organized as follows: The sample and experimental setup are presented in Sec. II. It is followed by the description of experimental results (Sec. III), as well as the drift-diffusion model and its assumptions (Sec. IV). Section V contains the discussion of the observed power and temperature dependences and their interpretation in terms of interplay between spin-orbit and hyperfine interaction. The conclusions are given in Sec. VI.

\section{SAMPLE AND EXPERIMENTAL SETUP}

The sample that we use in this work has already been studied in Refs. [11] and [13]. It contains a double quantum well (DQW) structure with two 12-nm-wide GaAs quantum wells separated by a 4-nm-wide $\mathrm{Al}_{0.45} \mathrm{Ga}_{0.55} \mathrm{As}$ barrier. An electric field is applied along the growth axis between a semitransparent 10 -nm-thick Ti top circular gate with a diameter of $300 \mu \mathrm{m}$ and $\mathrm{n}^{+}$-doped GaAs substrate, as depicted in Fig. 1(a). Figure 1(b) shows the band diagram of the biased DQW structure with direct exciton (DX) and IX transitions. A tunable titanium-sapphire laser is focused through a $\times 20$ objective (numerical aperture NA $=0.42$ ) to a beam of $3 \mu \mathrm{m}$ diameter at the sample surface. The sample is mounted inside an immersion $\mathrm{He}^{4}$ optical cryostat. The photoluminescence (PL) is collected through the same objective and split into two beams with orthogonal polarizations $\left(\sigma^{+}\right.$and $\left.\sigma^{-}\right)$, simultaneously imaged onto a spectrometer with magnification of 40. The spectrometer includes a CCD with a pixel size of $26 \mu \mathrm{m}$ such that the spatial imaging resolution is $0.65 \mu \mathrm{m}$. We use photoluminescence excitation (PLE) experiments to determine the energy of the DX state in our sample, $E_{D X}=$ $1.558 \mathrm{eV}$ (see Appendix). To optimize the spin pumping efficiency, we tune a $\sigma^{+}$-polarized excitation exactly to that energy. The excitation power is varied, resulting in variation of the resulting IX densities. The applied bias is fixed at $V_{g}=3 \mathrm{~V}$, corresponding to the IX emission energy $E=1.5408 \mathrm{eV}$ at the lowest measured temperature and excitation power. To determine the radiative lifetime $\tau_{r}$ of the IXs, we directly measured $\tau_{r}^{0}$ for a specific DX-IX energy separation $\Delta_{\mathrm{DX}-\mathrm{IX}}^{0}=$ $26 \mathrm{meV}$, using a time-resolved PL experiment. A radiative lifetime of $\tau_{r}^{0}=460 \mathrm{~ns}$ was found. To extract $\tau_{r}$ for all other experiments, we use the quadratic analytic model which was recently shown to be a good approximation for the dependence of $\tau_{r}$ on the DX-IX energy splitting $\Delta_{\mathrm{DX}-\mathrm{IX}}[11,27]$ :

$$
\tau_{r}=\tau_{r}^{0}\left(\frac{\Delta_{\mathrm{DX}-\mathrm{IX}}}{\Delta_{\mathrm{DX}-\mathrm{IX}}^{0}}\right)^{2} .
$$

\section{RESULTS}

Figure 2 shows typical color maps of a spatial cross section of PL spectra as a function of the distance from the excitation spot for two circular polarizations. These measurements are taken at three different excitation powers and three temperatures. For each pair of measurements $\sigma^{+} / \sigma^{-}$the intensity is normalized to its maximum in $\sigma^{+}$ polarization. Several observations could be made from these measurements: (i) The IX emission energy increases with increasing excitation power for each temperature. This is a signature of the increasing IX density. This energy shift results in the reduction of the IX lifetime $\tau_{r}$ [see Eq. (1) and Fig. 4(a)]. The low-energy shoulder in the emission could be associated with the presence of charged excitons in the system, characterized by an additional binding energy which matches the observed spectral splitting of about $1.5 \mathrm{meV}$. (ii) The IX emission is partially polarized, which means that the transfer between DX and IX states partially conserves the polarization, in agreement with previous reports [19-22,28]. (iii) There are variations in the spatial extent, polarization, and shape of the IX cloud for different excitation powers. This is a signature of the dipole-dipole interactions between IXs. There is a nontrivial dependence of the diffusion coefficient on the IX density and temperature due to an interplay between drift and diffusion currents of IX [29]. To get a closer look on the shape 

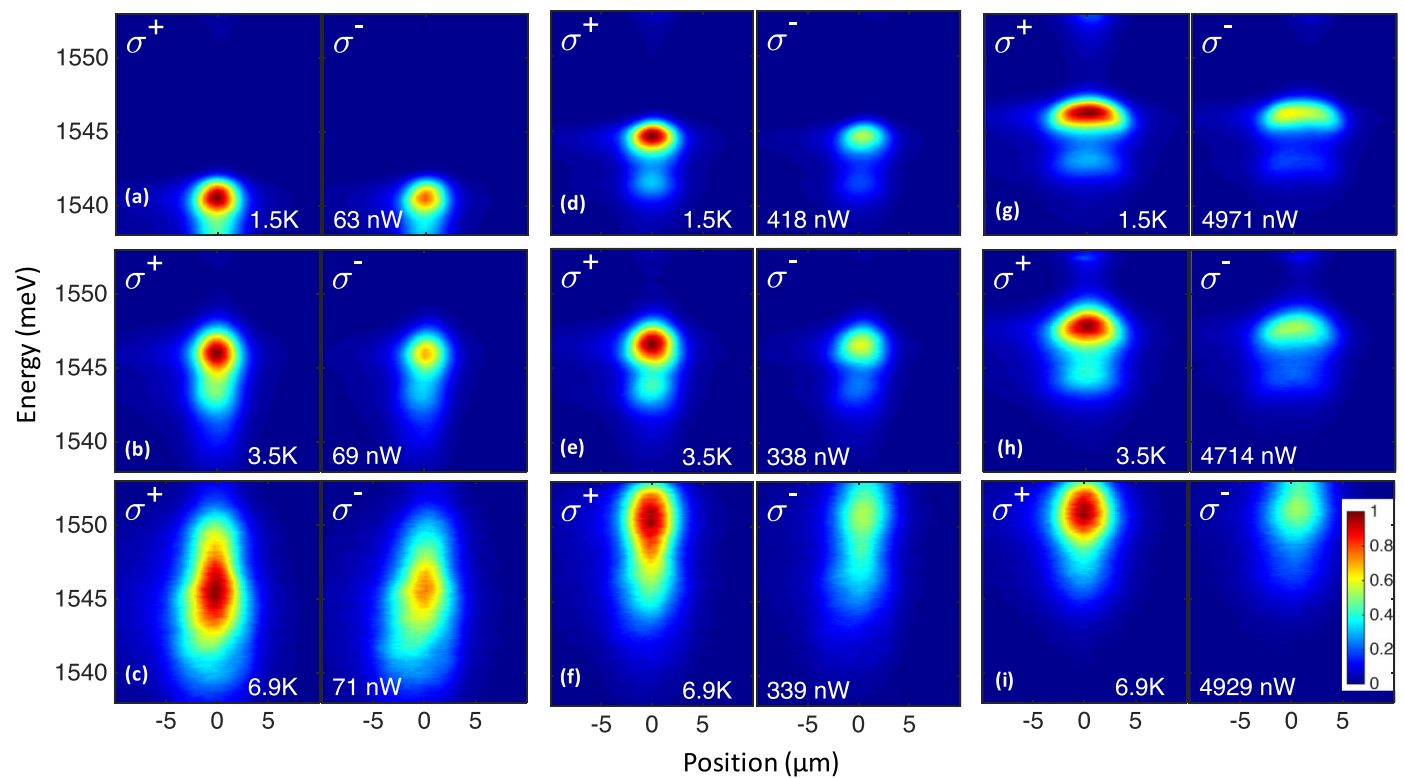

FIG. 2. Color-coded spatially resolved PL spectra of IXs in the two orthogonal circular polarizations. The measurements at three different excitation powers and three temperatures are shown. Each data set is normalized to its maximum in $\sigma^{+}$polarization.

of the IX emission intensity profile and its polarization, we plot in Fig. 3 the spectrally integrated intensity of the IX emission in $\sigma^{+}$(blue symbols) and $\sigma^{-}$(red symbols) polarizations for the same set of measurements as those shown in Fig. 2. The excitation spot size is shown by a black dashed line. To extract the IX spin lifetime and diffusivity from these data sets, we fit them to the drift-diffusion model presented in the next section.

\section{MODEL}

We consider the simplest model that accounts for radial drift, diffusion, and spin relaxation of IXs created by a circularly polarized pump beam with a $R_{0}$-radius Gaussian spot. The same model was used in Ref. [20].
Let $n=\left[n_{+2} n_{+1} n_{-1} n_{-2}\right]$ be the vector of IX densities in each of four IX spin states, and $D$ is the IX diffusion coefficient. IX mobility $\mu$ is related to the diffusion coefficient via the Einstein relation (linear transport regime [30]) $\mu=D / k_{B} T$, where $k_{B}$ is the Boltzmann constant and $T$ is the lattice temperature. The potential $u_{0}$ responsible for the drift of IXs out from the excitation spot is due to the dipole-dipole interaction between IXs [29-32]. In the simplest case $u_{0}$ is given by the plain capacitor formula $u_{0}=4 \pi^{2} d / \epsilon$, where $d$ is the distance between the quantum well centers and $\epsilon$ is the dielectric constant $[9,31,33,34]$. For the samples studied in this work, this yields $u_{0}=1.6 \times 10^{10} \mathrm{meV} \mathrm{\textrm {cm } ^ { 2 }}$. The exciton generation rate under $\sigma^{+1}$ excitation in the sample plane can be written as $\Lambda_{+1}=2\left(N_{p} / R_{0}^{2}\right) \exp \left(-r^{2} / R_{0}^{2}\right)$, where $r$ is the
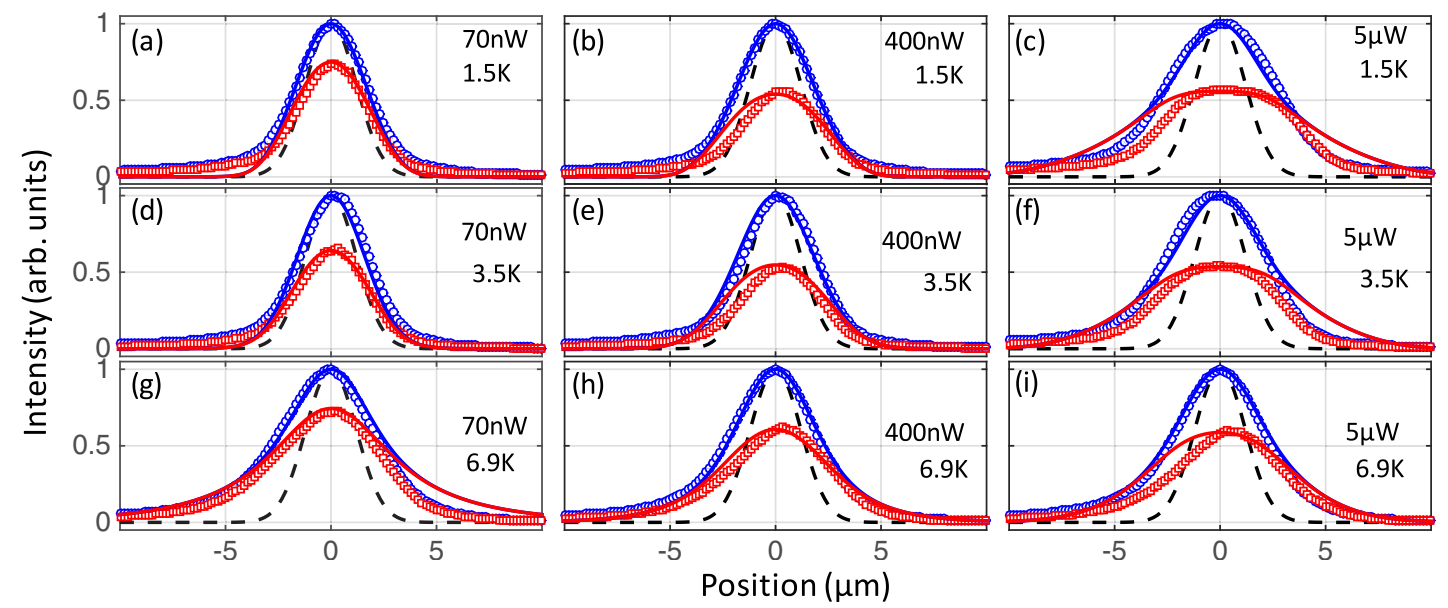

FIG. 3. Spatial distribution of IX emission intensity at several temperatures and excitation powers. $\sigma^{+}$(blue circles) and $\sigma^{-}$(red squares) intensity profiles are compared to the excitation spot profile (black dashed line). The solid lines are a fit to the model presented in Sec. IV. 
in-plane distance from the excitation spot center and $N_{p}$ is the number of excitons generated per second.

The drift-diffusion equation, including spin dynamics of the four exciton states, reads

$$
\frac{d n}{d t}=\nabla(D \nabla n)+\mu n \nabla\left[u_{0}\left(n_{b}+n_{d}\right)\right]+w n+\Lambda,
$$

$$
w=\left(\begin{array}{cc}
-\left(w_{e}^{+}+w_{h}^{+}\right) & w_{e}^{-} \\
w_{e}^{+} & -\left(\frac{1}{\tau_{r}}+w_{e}^{-}+w_{h}^{-}+w_{e x}\right) \\
w_{h}^{+} & w_{e x} \\
0 & w_{h}^{-}
\end{array}\right.
$$

$$
\begin{aligned}
2 \frac{d n_{-1}}{d t}= & 2 \nabla\left[D \nabla n_{-1}+2 \mu n_{-1} \nabla\left(u_{0} n_{b}\right)\right]-\frac{1}{\tau_{r}} n_{-1} \\
& -\frac{1}{\tau_{e}}\left(n_{-1}-n_{+1}\right) .
\end{aligned}
$$
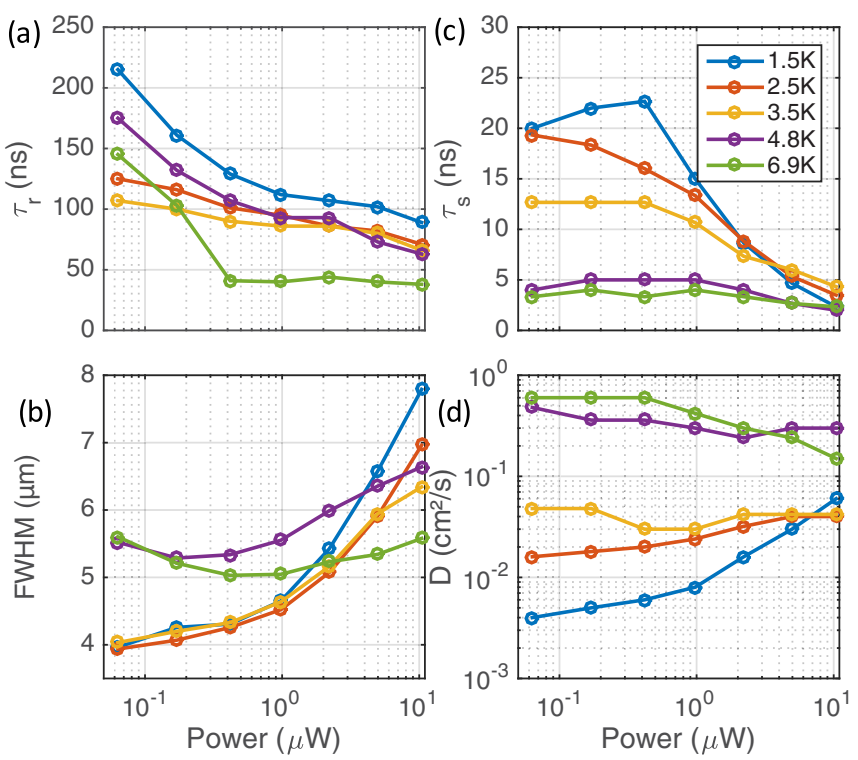

FIG. 4. (a) IX radiative lifetime $\tau_{r}$ used in the modeling, see Sec. II and Eq. (1). (b) IX cloud width at half maximum of intensity profile as a function of excitation power for several temperatures. IX spin lifetime $\tau_{s}$ (c) and exciton diffusion coefficient (d) are extracted from the drift-diffusion modeling of the PL spatial profiles, as those shown in Fig. 3. where $n_{b}+n_{d}=\left(n_{+2}+n_{+1}+n_{-1}+n_{-2}\right)$ is the total (bright and dark) exciton density and $\Lambda=\left[0 \Lambda_{+1} 00\right]$ is the generation rate vector. The exciton recombination and spin relaxation (including individual electron and hole spin flips, as well as the exciton spin flips) is represented by the matrix $w$ [35]:

$$
\left.\begin{array}{cc}
w_{h}^{-} & 0 \\
w_{e x} & w_{h}^{+} \\
-\left(\frac{1}{\tau_{r}}+w_{e}^{-}+w_{h}^{-}+w_{e x}\right) & w_{e}^{+} \\
w_{e}^{-} & -\left(w_{e}^{+}+w_{h}^{+}\right)
\end{array}\right) .
$$

Note that these equations do explicitly account for the dark excitons dynamics in the structure, but they assume that bright-dark conversion is infinitely fast, so that $n_{+2}=n_{-1}$ and $n_{-2}=n_{+1}$. One can see from Eqs. (4) and (5) that within the assumptions made here, the polarization lifetime (the time corresponding to the transition from one bright IX state to another) is simply equal to the electron spin-flip time $\tau_{s}=\tau_{e}$. In the following we refer to this time $\tau_{s}$ as the IX spin lifetime.

In order to find the steady-state solutions for the exciton density, we numerically solve this set of equations. The FWHM of the Gaussian excitation spot radius is extracted from the fit to the Gaussian function of the laser spot profile (dashed lines in Fig. 3).

When fitting the model to the experimental data, we assumed that the number of excitons generated per second $N_{p}$ increases linearly with the excitation power. At lowest power $P_{0}=70 \mathrm{nW}$ we have chosen $N_{p}^{0}=3 \times 10^{8} \mathrm{~s}^{-1}$. The value of $N_{p}^{0}$ is the first fitting parameter (actually, this value depends on the choice of $u_{0}$, the true fitting parameter is $N_{p}^{0} u_{0}$ ). The two other fitting parameters are the electron-spin relaxation time $\tau_{e}$ (or, equivalently, IX spin lifetime $\tau_{s}$ ) and the exciton diffusion constant $D$. The recombination time $\tau_{r}$ is determined for each set of data according to Eq. (1). The resulting values are shown in Fig. 4(a).

The comparison between the data and the calculated PL intensity (assumed to be proportional to the density of bright excitons) is shown in Fig. 3 for three different temperatures and powers. One can see in Figs. 4(c) and 4(d) that both $\tau_{s}$ and $D$ obtained within the fitting procedure appear to be temperature and power dependent. In the next section we discuss this dependence and the underlying physical mechanisms.

\section{DISCUSSION}

To better understand the spin dynamics of IXs, it is instructive to compare their spin lifetime to the diffusion constant, which determines the transport dynamics of the IX cloud. Both the spin and the transport show three distinct temperature regimes, as can be seen in Fig. 4.

(i) At high temperature, $T \geqslant 4.8 \mathrm{~K}$, the spin lifetime is rather short $\left(\tau_{s}<5 \mathrm{~ns}\right)$ and almost does not change with excitation power. At the same time, $D$ also remains approximately constant and the size of the cloud exhibits a weak, nonmonotonous behavior with power. The fact that $D$ 
is independent of excitation power is typical for a thermally activated cloud [30]. The nonmonotonous cloud size is due to the interplay between diffusion and drift of the excitons. It can be shown analytically that in the case of diffusiondominated transport, the FWHM of the cloud does not depend on $N_{p}$, the number of particles injected in the system per unit time, but only on $\left(D \tau_{r}\right)^{1 / 2}$. By contrast, in the case of drift-dominated transport, the FWHM of the cloud increases as $\left(D N_{p} / k_{B} T\right)^{1 / 4} \tau_{r}^{1 / 2}$. The nonmonotonous behavior of the cloud size when excitation power increases could thus be the signature of transition between these two regimes [29,32,36].

(ii) A very different behavior is seen in the lower temperature regime, $1.5 \mathrm{~K}<T<4.8 \mathrm{~K}$. The spin lifetime sharply rises as the temperature decreases, reaching an almost a fivefold increase at $2.5 \mathrm{~K}$. Spin lifetime also exhibits a strong power dependence, with a clear threshold power of $\simeq 0.5 \mu \mathrm{W}$, above which it strongly decreases. This trend of $\tau_{s}$ is clearly anticorrelated with both the size of the cloud and the diffusion constant obtained from the modeling. Indeed, the diffusion constant $D$ sharply decreases with decreasing temperature in this regime. As for the power dependence, $D$ is approximately constant with increasing power up to the same threshold of $\simeq$ $0.5 \mu \mathrm{W}$ and increases only above this threshold. The IX cloud does not show any expansion up to a similar excitation power, and then experience a strong expansion. Such anticorrelation of $\tau_{s}$ and $D$ is well explained within the DP mechanism, where the IX spin-relaxation time $\tau_{s}$ reads

$$
\tau_{s, S O}^{-1}=\Omega_{S O}^{2} \tau_{p},
$$

where $\tau_{p}$ is the momentum scattering time, $\Omega_{S O}=2 \beta k$ is the electron-spin precession frequency in the presence of an effective magnetic field due to spin-orbit interaction, $\beta$ is the spin-orbit interaction constant, $k=\sqrt{2 m_{\mathrm{exc}} k_{B} T / \hbar^{2}} m_{e} / m_{\mathrm{exc}}$ is the thermal wave vector of an electron within the exciton, and $m_{e}\left(m_{\mathrm{exc}}\right)$ is the electron (exciton) mass. Because $\tau_{p}=$ $D m_{\text {exc }} / k_{B} T$, an increase of the diffusion constant leads to a decrease of the spin relaxation time, as has been reported by Leonard et al. [20]. Note, however, that the persistent localization of the IX cloud below $T=4.8 \mathrm{~K}$ over a very large range of the excitation powers is consistent with previous reports of a transition into a spatially localized dark liquid of IX $[12,13]$, which was interpreted as a condensation into a dark quantum liquid of dark spin states $[11,13,14,17]$. In particular, Cohen et al. observed a similar power threshold over which there is a strong increase in IX mobility and the IX cloud expansion [13,14].

(iii) Focusing on the lowest measured temperature of $1.5 \mathrm{~K}$ (which also corresponds to the temperature where the transition to the liquid phase in Ref. [13] was found to be fully established), a very distinct behavior of $\tau_{s}$ with power is observed. In this regime a clear increase of $\tau_{s}$ when the power increases is seen, together with a slow increase in $D$. This correlation between $D$ and $\tau_{s}$ continues up to $\simeq 0.5 \mu \mathrm{W}$, after which a strong increase of $D$ is observed together with a strong decrease of $\tau_{s}$, where the previously discussed anticorrelation between $\tau_{s}$ and $D$ is established again. This low-power regime of correlation cannot be understood within DP mechanism and suggests that the hyperfine interaction with the nuclear spins of the GaAs lattice is involved in the spin relaxation process $[4,6,7]$. Indeed, the fluctuating Overhauser field $B_{N}$ created by the nuclear spins induces a spin relaxation given by a formula very similar to the one corresponding to the DP mechanism,

$$
\tau_{s, N}^{-1}=\frac{1}{3} \Omega_{N}^{2} \tau_{c}
$$

where $\Omega_{N}=g \mu_{B} B_{N}$ is the electron-spin precession frequency due to the fluctuating Overhauser field, $\tau_{c}$ is the correlation time of this field, and $g$ is the electron $g$ factor. The correlation time $\tau_{c}$ is related to the exciton diffusion coefficient and can be estimated as the time over which an exciton moves by a distance corresponding to its own radius $a_{\mathrm{exc}}: \tau_{c}=a_{\mathrm{exc}}^{2} / D$. In contrast to the spin relaxation induced by spin-orbit interaction, hyperfine-induced spin relaxation is enhanced when exciton diffusion slows down. Therefore, this relaxation mechanism could explain the initial increase of $\tau_{s}$ with power, as observed at $T=1.5 \mathrm{~K}$.

This spin-relaxation mechanism was not observed to date in any excitonic system. It stems from the very-low-mobility regime achieved for the IX cloud, allowing for the hyperfine interaction induced dephasing to become dominant. As the power increases, there is a transition from the hyperfine interaction to the DP dephasing mechanism, corresponding to the sharp increase in the IX diffusivity. While localization of excitons in the quantum well may arise due to disorder, i.e., monolayer fluctuations in the quantum well width, we note that the temperature and power regimes where strong IX fluid localization and hyperfine-induced spin relaxation is observed in this work seem to overlap with the regime where the IX liquid formation was observed in Ref. [13]. We thus can speculate that the reason for this strong localization of the IX cloud could be the formation of a dark IX liquid, similar to the previous reports [11-14,17]. If this is indeed the case, it shows that measuring spin polarization can be a complementary, sensitive tool for mapping the collective states of IX fluids.

In order to get a unified picture of the observed phenomena, we write the total spin relaxation time, due to both hyperfine and spin-orbit relaxations, as

$$
\tau_{s}^{-1}=\tau_{s, N}^{-1}+\tau_{s, S O}^{-1},
$$

where $\tau_{s}$ clearly has a nonmonotonous dependence on the diffusion constant. Figure 5 shows $\tau_{s}(D)$ as calculated from Eq. (8) (solid line) or extracted from measurements fitted by the drift-diffusion model of Eqs. (4) and (5) (symbols). We assume $B_{N}=b_{N} / \sqrt{N_{N}}$, where $b_{N}=5.3 \mathrm{~T}$ is the nuclear field at saturation [3] and $N_{N} \simeq 10^{5}$ is the number of nuclei within the exciton volume $V_{\mathrm{exc}}=\pi a_{\mathrm{exc}}^{2} L$, where $L$ is the quantum well width. The $g$-factor of the $L=12 \mathrm{~nm} \mathrm{GaAs}$ quantum well is $g \simeq 0.35$ [5], and the spin-orbit constant $\beta=25 \mathrm{meV} \AA$ [20]. The theoretical curve $\tau_{s}(D)$ has a nonmonotonous dependence and reaches a maximum $\tau_{s}^{\max }=\sqrt{3 / 8} v_{t h} /\left(\Omega_{S O} \Omega_{N} a_{\mathrm{exc}}\right)$ at $D^{\max }=\left(\Omega_{N} / \Omega_{S O}\right) a_{\mathrm{exc}} v_{t h} / \sqrt{6}$, where $v_{t h}=\sqrt{2 k_{B} T / m_{\mathrm{exc}}}$ is the thermal velocity of excitons. Remarkably, this curve does not depend on temperature, because $\Omega_{S O}$ and $v_{t h}$ have the same temperature dependence. Numerically one finds $\tau_{s}^{\max } \simeq 35 \mathrm{~ns}$ and $D^{\max } \simeq 3.7 \times 10^{-3} \mathrm{~cm}^{2} / \mathrm{s}$, close to the experimental observation. The theoretical curve being universal, it can be directly compared to the experimental data extracted from 


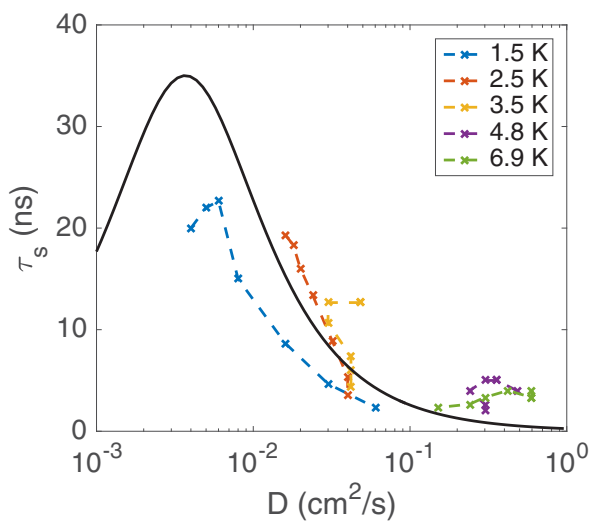

FIG. 5. IX spin lifetime as a function of the diffusion constant as extracted from the drift-diffusion modeling. Black line is the theoretical estimation based on Eq. (8).

the numerical modeling at any temperature. Since there are no fitting parameters in the theory, the agreement can be considered as satisfactory.

\section{CONCLUSIONS}

To conclude, our analysis suggests that IX spin lifetime is limited not only by the spin-orbit interaction via the DP mechanism, but also by the hyperfine interaction with the spins of the lattice nuclei. The crossover between these two different regimes corresponds to a transition from a highmobility state of the fluid to a very-low-mobility state, and may be correlated with the power and temperature regime where a condensation of a dipolar IX fluid was previously reported. These results suggest that the IX spin polarization could be a sensitive supplementary probe for the onset of the collective states in dipolar fluids of excitons. Further work, both theoretical and experimental, is required to confirm the relation between spin dynamics and collective properties of IX liquids. In particular, it would be instructive to combine the constant energy scheme $\left(E_{D X}-E_{I X}=\right.$ const $)$ used in Ref. [13] with resonant spin-polarized excitation used in this work to achieve better control over bright and dark exciton densities. Theoretically, the simplified model that we used must be extended to the case of nonthermal distribution of the IX population between bright and dark states. The assumption of the infinitely fast hole spin relaxation must be lifted in this case. The comparison between such a model and the full set of data obtained within the constant energy scheme will settle definitively the origin of exciton localization and the onset of the hyperfine-induced spin relaxation.

\section{ACKNOWLEDGMENTS}

We would like to acknowledge financial support from the German Deutsche Forschungsgemeinschaft (Grant No. SA-598/9), from the German Israeli Foundation (Grant No. GIF I-1277-303.10/2014), from the Israeli Science Foundation (Grant No. 1319/12), and the French National Research Agency (Grant OBELIX, No. ANR-15-CE30-0020-02). The work at Princeton University was funded by the Gordon and Betty Moore Foundation through the Emergent Phenomena in
Quantum Systems initiative (Grant No. GBMF4420), and by the National Science Foundation Materials Research Science and Engineering Center (Grant No. DMR-1420541).

\section{APPENDIX}

\section{Dependence on excitation energy}

In order to determine the resonant energy levels of the DQW structure, a photoluminescence excitation (PLE) experiment was conducted in the following way: a tunable $\mathrm{cw}$ titanium-sapphire laser was scanned through a wavelength range covering the expected optical direct exciton resonances while monitoring the (e1:hh1) 1S IX PL energy, intensity, and polarization.

Figure 6 shows the results of a polarization-resolved PLE experiment. Figures 6(a) and 6(b) show the integrated intensity and the peak energy of IX as a function of the excitation energy of the laser. There are two distinct peaks, separated by about $14 \mathrm{meV}$ in energy. We attribute the high-energy peak to the (e1:lh1) 1S DX absorption line and the lower one to the (e1:hh1) 1S DX. A large blueshift of the IX line is observed when the excitation is at resonance with one of the DX absorption lines. This is not surprising, as it is expected that the DX resonances would have a large absorption cross section and therefore an efficient conversion of photons to IX population, by carrier tunneling to adjacent quantum wells.

Figure 6(c) presents the degree of circular polarization (DOP) of IX as a function of the excitation energy. It can be seen that the DOP of the IX is maximal for laser excitation
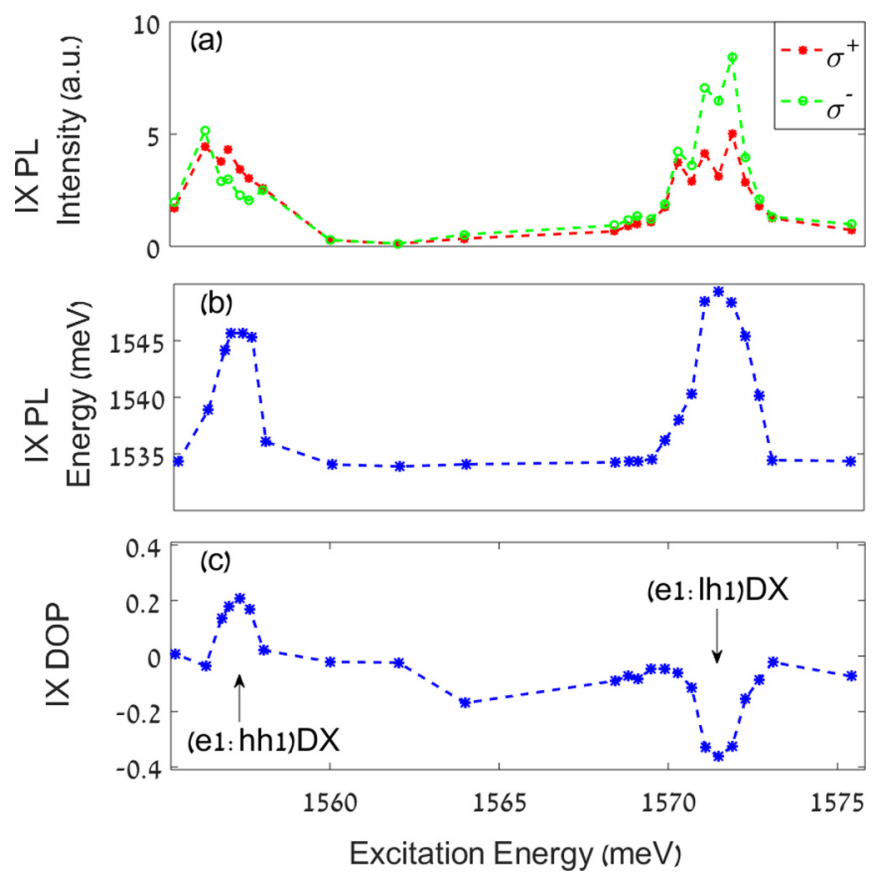

FIG. 6. Polarization-resolved PLE of IX. (a) The integrated PL intensity, monitored at the IX line for both polarizations. (b) The PL energy and (c) the degree of circular Polarization (DOP). A negative DOP means that the PL of the IX has the opposite circular polarization with respect to the circularly polarized exciting laser. The laser power here is $1.3 \mu \mathrm{W}$ and is circularly polarized with $\sigma^{+}$. The energies of (e1:hh1) DX and (e1:lh1) DX are pointed in (c) as a guide to the eye. 
resonant with the DX transitions. While for excitation at the (e1:hh1) DX transition, IXs are polarized in parallel to the exciting laser (positive DOP), an opposite polarization appears when the laser is resonant with the (e1:lh1) DX transition (negative DOP). This could be explained by the following mechanism: A resonant excitation with $\sigma^{+}$polarized light at the $(\mathrm{e} 1: \ln 1)$ line creates the following exciton state: $\left|\frac{1}{2}, \frac{1}{2}\right\rangle$. The light-hole in the exciton rapidly relaxes to the heavy-hole subband where it could be either at $\left|\frac{1}{2}, \frac{3}{2}\right\rangle$ state, which is dark, or at $\left|\frac{1}{2},-\frac{3}{2}\right\rangle$ state, which is bright and has $S_{z}=-1$.
Thus an initial population of IX with polarization opposite to the laser is created. From here on, the IX population follows the spin-relaxation mechanism. Such "opposite" initial polarization was observed in single GaAs quantum well by time-resolved experiments but not to this extent [37]. Another interesting feature is that the density and the DOP following a (e1:lh1) DX excitation seem to be larger than at a (e1:hh1) DX excitation. The increase in density is in contrast with the known oscillator strength ratios in bulk GaAs, which is 3 times larger for the (e1:hh1) transition than the (e1:lh1) transition [38].
[1] J. Wunderlich, B. Kaestner, J. Sinova, and T. Jungwirth, Phys. Rev. Lett. 94, 047204 (2005).

[2] J. D. Koralek, C. P. Weber, J. Orenstein, B. A. Bernevig, S.-C. Zhang, S. Mack, and D. D. Awschalom, Nature (London) 458, 610 (2009).

[3] Optical Orientation, edited by F. Meier and B. Zakharchenya, Modern Problems in Condensed Matter Science Series Vol. 8 (North-Holland, Amsterdam, 1984).

[4] K. V. Kavokin, Semicond. Sci. Technol. 23, 114009 (2008).

[5] Spin Physics in Semiconductors, edited by M. I. Dyakonov, Springer Series in Solid-State Sciences (Springer, Berlin, 2008).

[6] R. I. Dzhioev, K. V. Kavokin, V. L. Korenev, M. V. Lazarev, B. Y. Meltser, M. N. Stepanova, B. P. Zakharchenya, D. Gammon, and D. S. Katzer, Phys. Rev. B 66, 245204 (2002).

[7] Z. Chen, S. G. Carter, R. Bratschitsch, P. Dawson, and S. T. Cundiff, Nat. Phys. 3, 265 (2007).

[8] M. Combescot, O. Betbeder-Matibet, and R. Combescot, Phys. Rev. Lett. 99, 176403 (2007).

[9] B. Laikhtman and R. Rapaport, Europhys. Lett. 87, 27010 (2009).

[10] Y. E. Lozovik, I. L. Kurbakov, and I. V. Ovchinnikov, Solid State Commun. 126, 269 (2003).

[11] Y. Shilo, K. Cohen, B. Laikhtman, K. West, L. Pfeiffer, and R. Rapaport, Nat. Commun. 4, 2335 (2013).

[12] M. Stern, V. Umansky, and I. Bar-Joseph, Science 343, 55 (2014).

[13] K. Cohen, Y. Shilo, K. West, L. Pfeiffer, and R. Rapaport, Nano Lett. 16, 3726 (2016).

[14] Y. Mazuz-Harpaz, K. Cohen, and R. Rapaport, Superlattices Microstruct. 108, 88 (2017).

[15] A. A. High, J. R. Leonard, A. T. Hammack, M. M. Fogler, L. V. Butov, A. V. Kavokin, K. L. Campman, and A. C. Gossard, Nature (London) 483, 584 (2012).

[16] A. A. High, J. R. Leonard, M. Remeika, L. V. Butov, M. Hanson, and A. C. Gossard, Nano Lett. 12, 2605 (2012).

[17] M. Alloing, M. Beian, M. Lewenstein, D. Fuster, Y. González, L. González, R. Combescot, M. Combescot, and F. Dubin, Europhys. Lett. 107, 10012 (2014).

[18] A. A. High, A. T. Hammack, J. R. Leonard, S. Yang, L. V. Butov, T. Ostatnický, M. Vladimirova, A. V. Kavokin, T. C. H. Liew, K. L. Campman, and A. C. Gossard, Phys. Rev. Lett. 110, 246403 (2013).

[19] A. Violante, R. Hey, and P. V. Santos, Phys. Rev. B 91, 125302 (2015).

[20] J. R. Leonard, Y. Y. Kuznetsova, S. Yang, L. V. Butov, T. Ostatnický, A. Kavokin, and A. C. Gossard, Nano Lett. 9, 4204 (2009).
[21] K. Kowalik-Seidl, X. P. Vögele, B. N. Rimpfl, S. Manus, J. P. Kotthaus, D. Schuh, W. Wegscheider, and A. W. Holleitner, Appl. Phys. Lett. 97, 011104 (2010).

[22] M. Beian, M. Alloing, E. Cambril, C. G. Carbonell, J. Osmond, A. Lemaître, and F. Dubin, Europhys. Lett. 110, 27001 (2015).

[23] P. Andreakou, S. Cronenberger, D. Scalbert, A. Nalitov, N. A. Gippius, A. V. Kavokin, M. Nawrocki, J. R. Leonard, L. V. Butov, K. L. Campman, A. C. Gossard, and M. Vladimirova, Phys. Rev. B 91, 125437 (2015).

[24] P. Andreakou, A. V. Mikhailov, S. Cronenberger, D. Scalbert, A. Nalitov, A. V. Kavokin, M. Nawrocki, L. V. Butov, K. L. Campman, A. C. Gossard, and M. Vladimirova, Phys. Rev. B 93, 115410 (2016).

[25] A. Vinattieri, J. Shah, T. C. Damen, K. W. Goossen, L. N. Pfeiffer, M. Z. Maialle, and L. J. Sham, Appl. Phys. Lett. 63, 3164 (1993).

[26] M. I. D’yakonov and V. Perel, Zh. Eksp. Teor. Fiz 33, 1954 (1971).

[27] Y. Mazuz-Harpaz, K. Cohen, B. Laikhtman, R. Rapaport, K. West, and L. N. Pfeiffer, Phys. Rev. B 95, 155302 (2017).

[28] M. Poggio, G. M. Steeves, R. C. Myers, N. P. Stern, A. C. Gossard, and D. D. Awschalom, Phys. Rev. B 70, 121305(R) (2004).

[29] R. Rapaport, G. Chen, and S. H. Simon, Phys. Rev. B 73, 033319 (2006).

[30] R. Rapaport, G. Chen, S. Simon, O. Mitrofanov, L. Pfeiffer, and P. M. Platzman, Phys. Rev. B 72, 075428 (2005).

[31] A. L. Ivanov, Europhys. Lett. 59, 586 (2002).

[32] R. Rapaport and G. Chen, J. Phys.: Condens. Matter 19, 295207 (2007).

[33] C. Schindler and R. Zimmermann, Phys. Rev. B 78, 045313 (2008).

[34] M. Remeika, J. R. Leonard, C. J. Dorow, M. M. Fogler, L. V. Butov, M. Hanson, and A. C. Gossard, Phys. Rev. B 92, 115311 (2015).

[35] M. Z. Maialle, E. A. de Andrada e Silva, and L. J. Sham, Phys. Rev. B 47, 15776 (1993).

[36] Z. Vörös, R. Balili, D. W. Snoke, L. Pfeiffer, and K. West, Phys. Rev. Lett. 94, 226401 (2005).

[37] B. Dareys, X. Marie, T. Amand, J. Barrau, Y. Shekun, I. Razdobreev, and R. Planel, Superlattices Microstruct. 13, 353 (1993).

[38] P. Y. Yu and M. Cardona, Fundamentals of Semiconductors, Graduate Texts in Physics (Springer, Berlin, 2010). 